\title{
Electrically tunable charge and spin transitions in Landau levels of interacting Dirac fermions in trilayer graphene
}

\author{
Vadim M. Apalkov \\ Department of Physics and Astronomy, Georgia State University, Atlanta, Georgia 30303, USA \\ Tapash Chakraborty \\ Department of Physics and Astronomy, University of Manitoba, Winnipeg, Canada R3T 2N2
}

(Dated: October 11, 2018)

\begin{abstract}
Trilayer graphene in the fractional Quantum Hall Effect regime displays a set of unique interactioninduced transitions that can be tuned entirely by the applied bias voltage. These transitions occur near the anti-crossing points of two Landau levels. In a large magnetic field ( $>8 \mathrm{~T}$ ) the electronelectron interactions close the anti-crossing gap, resulting in some unusual transitions between different Landau levels. For the filling factor $\nu=\frac{2}{3}$, these transitions are accompanied by a change of spin polarization of the ground state. For a small Zeeman energy, this provides an unique opportunity to control the spin polarization of the ground state by fine tuning the bias voltage.
\end{abstract}

Dirac fermions in monolayer and bilayer graphene with their remarkable electronic properties have received extraordinary scrutiny in recent years [2, 3]. In an external magnetic field, these systems exhibit unconventional quantum Hall effects [4, 5] that are direct manifestations of their rather unusual band structures [6, 7]. As a consequence, the Landau level (LL) energies of these systems are very different from those of conventional two-dimensional electrons systems (2DESs). More specifically, in monolayer graphene the Landau level energies exhibit a square root dependence on the applied field [8], while in bilayer graphene one finds a linear dependence [9]. On the other hand, interactions among Dirac fermions in the fractional quantum Hall effect (FQHE) 10,11] regime reveals several rather unexpected and intriguing effects in monolayer [12] and bilayer [13] graphene. Recent experimental observation of the FQHE in monolayer graphene [14, 15] have indeed confirmed the important role electron-electron interactions play in these systems. Clearly, the dynamics of Dirac fermions are sensitive to the number of graphene layers present in the system and their stacking arrangements. Quite expectedly, the attention has now shifted to the investigation of the electronic properties of Dirac fermions in trilayer graphene (TLG).

A TLG consisting of three coupled graphene layers has a very unique electronic energy spectrum. Within the nearest-neighbor inter-layer coupling approximation the energy spectrum of TLG with Bernal stacking consists effectively of decoupled single-layer graphene and the bilayer graphene energy spectra. Therefore the TLG allows us to study the energy spectra of both the massless and massive Dirac fermions within a single system. In a strong perpendicular magnetic field, the LL energy spectrum of TLG becomes a combination of Landau levels of single-layer and bilayer graphene [16]. The spectrum exhibits many crossings of the Landau levels as a function of the magnetic field. At the crossing points the Landau levels are highly degenerate. The degeneracy is lifted when higher-order inter-layer coupling terms are taken
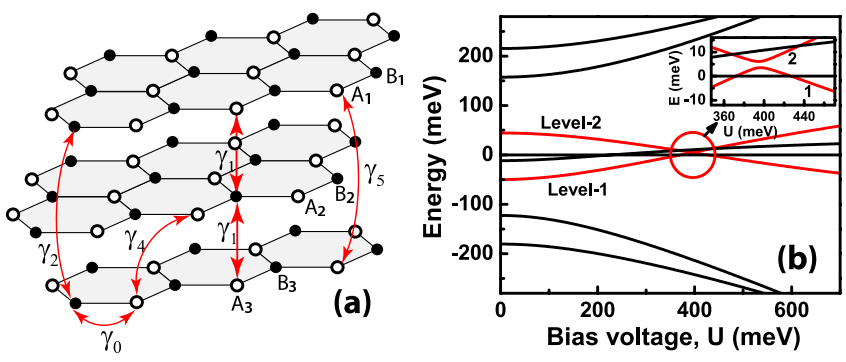

FIG. 1: (a) Schematic illustration of the ABA stacking of TLG. Each graphene layer consists of two inequivalent sites A and B. The inter-layer and intra-layer hopping integrals, $\gamma_{i}$, show the couplings, which are included in the single-particle Hamiltonian (1). (b) Landau level energy spectrum of TLG in a magnetic field of $15 \mathrm{~T}$ as a function of the bias voltage, $U$. Two red lines show anti-crossing for $U \approx 400 \mathrm{meV}$. The corresponding LLs are labelled as level-1 and level-2, respectively. The LLs 1 and 2 belong to the set of Landau levels with parameter $n=0$. The inset shows the region of anti-crossing. The anti-crossing gap is $\approx 2.6 \mathrm{meV} \approx 30 \mathrm{~K}$.

into account, resulting in several unusual properties of the quantum Hall effect in trilayer graphene [17].

While the single-particle features of the quantum Hall effect are interesting, here we show that by introducing interactions among the Dirac fermions in a TLG in the FQHE regime, we witness several very unique properties of the TLG that goes far beyond the mere level crossings observed in the integer QHE. We found several LL repulsions and level crossings which resulted in some interesting spin transitions among the LLs in this system that have no analogues in the interaction-induced spinreversed ground states and elementary excitations discovered earlier in conventional electron systems [18, 19]. These spin transitions in the TLG are driven by an applied perpendicular bias field for a fixed magnetic field, and therefore we expect these novel transitions to be entirely tunable.

In what follows, we only consider the Bernal or ABAstacking of our TLG. In the tight-binding approximation 
the Hamiltonian of TLG is characterized by the intralayer hopping integral, $\gamma_{0}=3.1 \mathrm{eV}$, and inter-layer hopping integrals, $\gamma_{1}=0.39 \mathrm{eV}, \gamma_{2}=-0.028 \mathrm{eV}, \gamma_{4}=0.041$ $\mathrm{eV}$, and $\gamma_{5}=0.05 \mathrm{eV}$, corresponding to different types of the inter-layer coupling, shown schematically in Fig.
1 (a) [17]. In the basis $\left(\psi_{A_{1}}-\psi_{A_{3}}, \psi_{B_{1}}-\psi_{B_{3}}, \psi_{A_{1}}+\right.$ $\left.\psi_{A_{3}}, \psi_{B_{2}}, \psi_{A_{2}}, \psi_{B_{1}}+\psi_{B_{3}}\right)$ and in a perpendicular magnetic field, the Hamiltonian of a TLG for a single valley, e.g., valley $K$, takes the form [16, 17]

$$
\mathcal{H}=\left(\begin{array}{cccccc}
-\gamma_{2} / 2 & v_{0} \pi_{+} & -U / 2 & 0 & 0 & 0 \\
v_{0} \pi_{-} & -\gamma_{5} / 2+\delta & 0 & 0 & 0 & -U / 2 \\
-U / 2 & 0 & +\gamma_{2} / 2 & 0 & -\sqrt{2} v_{4} \pi_{+} & v_{0} \pi_{+} \\
0 & 0 & 0 & 0 & v_{0} \pi_{-} & -\sqrt{2} v_{4} \pi_{-} \\
0 & 0 & -\sqrt{2} v_{4} \pi_{-} & v_{0} \pi_{+} & \delta & \sqrt{2} \gamma_{1} \\
0 & -U / 2 & v_{0} \pi_{-} & -\sqrt{2} v_{4} \pi_{+} & \sqrt{2} \gamma_{1} & \gamma_{5} / 2+\delta
\end{array}\right)
$$

where $v_{0}=(\sqrt{3} / 2) a \gamma_{0} / \hbar \approx 10^{6} \mathrm{~m} / \mathrm{s}, v_{4}=(\sqrt{3} / 2) a \gamma_{4} / \hbar$, and $\pi_{ \pm}=\pi_{x} \pm \pi_{y}$. Here $\vec{\pi}=\vec{p}+e \vec{A} / c$ is the generalized momentum. The parameter $\delta=0.046 \mathrm{eV}$ is the difference between the on-site energies of two sublattices within a single graphene layer [17]. The bias voltage, $U$, is introduced in the Hamiltonian (11) as the potential difference, i.e., the on-site energy difference, between layers 1 and 3 . Here we assume that the potential of layer 1 is zero, while the potential of layer 2 and 3 are $U / 2$ and $U$, respectively. The bias voltage, $U$, is considered as the parameter of the system, which can be varied externally.

The LLs of a TLG can be obtained from the Hamiltonian matrix (11). The corresponding wave functions are parametrized by the integer $n$ and can be expressed through the conventional (non-relativistic) Landau level wave functions, $\phi_{n, m}$ as

$$
\Psi=\left(\begin{array}{c}
C_{1} \phi_{n+2, m} \\
C_{2} \phi_{n+1, m} \\
C_{3} \phi_{n+2, m} \\
C_{4} \phi_{n, m} \\
C_{5} \phi_{n+1, m} \\
C_{6} \phi_{n+1, m}
\end{array}\right)
$$

where $m$ is the intra-Landau level parameter, e.g., the angular momentum, and $C_{i}$ are constants. Therefore, the LL wave functions of a TLG are combinations of $n$, $n+1$, and $n+2$ non-relativistic Landau functions.

In what follows, we consider a many-electron system partially occupying a single LL of the TLG. We study the properties of these systems in the FQHE regime, specifically for the filling factors, $\nu=\frac{1}{3}, \frac{2}{3}$, and $\frac{2}{5}$. In these cases, the conventional non-relativistic system shows incompressible behavior with a finite energy gap 10, 11. The interaction properties of the many-electron system occupying a single Landau level are completely determined by the Haldane pseudopotentials $V_{m}^{(n)}$ [20], which are the interaction energies of two electrons with relative angular momentum, $m$. They are determined by making use of the LL wave functions (2) [21]. We numerically evaluate the FQHE state in a TLG by considering a finite-size system of $N$ electrons in a spherical geometry [20] with interaction potentials determined by the Haldane pseudopotentials. The radius of the sphere is $\sqrt{S} \ell_{0}$, where $2 S$ is the number of magnetic fluxes through the sphere in units of the flux quantum, and $\ell_{0}=\sqrt{e \hbar / c B}$ is the magnetic length. The parameter $S$ also determines the number of single-particles states, $2 S+1$, and for finite number of electrons - the filling factor of the system.

From the Hamiltonian (1) we numerically evaluate the single-particle LL energy spectrum. The TLG LLs are parametrized by the integer $n$ [see Eq. (2)]. For each $n$ there are 6 LLs in a TLG. A typical LL spectrum is shown in Fig. 1 (b). The spectrum as a function of the bias voltage (or the magnetic field) shows crossing and anti-crossing of the energy levels. The anti-crossing gap, shown in Fig. 1 (b), is around $2.6 \mathrm{meV} \approx 30 \mathrm{~K}$ for a magnetic field of 15 Tesla. Below we show that the FQHE in TLG has non-trivial and unique interactioninduced properties near these anti-crossing points. We study the behavior of the system near the special anticrossing point shown as inset in Fig. 1 (b). This point corresponds to anti-crossing of the TLG LLs with $n=0$. We label the corresponding levels as Landau level-1 (LL1) and Landau level-2 (LL-2) [Fig. 1 (b)] and consider the FQHE states only in these levels. For each FQHE state in the corresponding LL-1 and LL-2 we have evaluated the ground state energy per particle and the excitation gap for the incompressible states.

We first consider the fundamental $\nu=\frac{1}{3}$-FQHE states in LLs 1 and 2, respectively [Fig. 2]. The system behaves very differently for weak and strong magnetic fields. In a weak magnetic field $(B=5 \mathrm{~T})$ the many-particle states show anti-crossing (Fig. 2a) similar to the singleparticle levels [Fig. 1 (b)]. This anti-crossing is clearly visible in the dependence of the $\frac{1}{3}$-FQHE gaps since the values of the FQHE gaps in LL-1 and LL-2 are interchanged when the system goes through the anti-crossing point. The system shows an interesting behavior exactly 


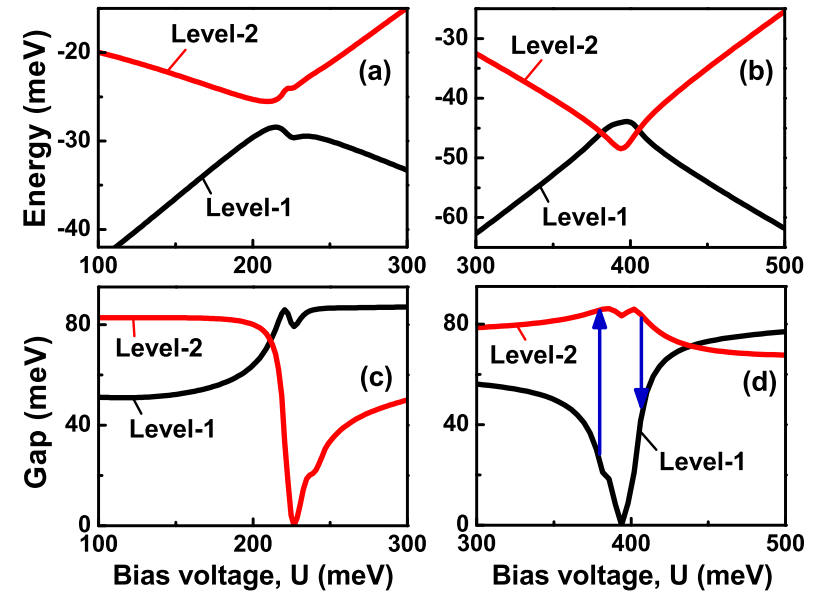

FIG. 2: Numerical results for finite-size $\nu=\frac{1}{3}$-FQHE systems in LL 1 and LL 2, shown as a function of the bias voltage near the anti-crossing points. The magnetic field is 5 Tesla [panels (a) and (c)] and 15 Tesla [panels (b) and (b)]. Panels (a) and (b) show the ground state energy per particle, while panels (c) and (d) show the excitation gaps at the corresponding energy levels with filling factor $\nu=\frac{1}{3}$. Blue arrows in panel (d) indicate jumps of the FQHE gaps at the level crossing in panel (b). The number of electrons is $N=9$ and the parameter of the sphere is $2 S=24$.

at the anti-crossing point. Here, due to a mixture of the single-particle wavefunctions of LL-1 and LL-2, the many-particle interaction properties are enhanced in LL1 while suppressed in LL-2. As a result, at the anticrossing point, the $\frac{1}{3}$-FQHE gap in LL-1 has a maximum while the $\frac{1}{3}$-state in LL-2 becomes compressible with a vanishing gap [Fig. 2 (c)]. Due to a larger cohesive energy of the incompressible state compared to the compressible one, the many-particle anti-crossing gap shows a small enhancement relative to the single-particle value by 0.4 $\mathrm{meV} \approx 5 \mathrm{~K}$. Experimentally, the anti-crossing properties of TLG in a small magnetic field can be observed by studying the FQHE in LL-2. In such a system, with increasing bias voltage one would observe a transition FQHE - no FQHE - FQHE within a single LL, just as we predicted earlier for bilayer graphene [13].

Near the anti-crossing point in a large magnetic field $(B=15 \mathrm{~T})$, TLG shows several novel features [Fig.2 $(\mathrm{b}, \mathrm{d})]$ : The anti-crossing of the single-particle energy levels becomes double crossings for the many-particles states. This means that the cohesive energy of the manyparticle state in LL-2 is larger than that in LL-1, and this difference overcomes the anti-crossing gap. The reason for such a behavior is the change in the interaction strength in LL-1 and LL-2. For a large magnetic field the many-particle interaction potential at the anticrossing point becomes stronger in LL-2 and weaker in LL-1, which is opposite to what we see for a weak magnetic field [Fig. $2(\mathrm{a}, \mathrm{c})]$. As a result, the FQHE gap in

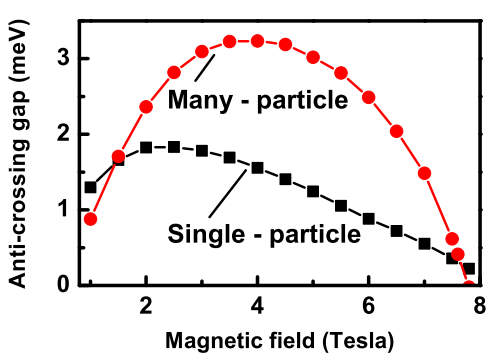

FIG. 3: The anti-crossing gap corresponding to the anticrossing of LL-1 and LL-2 [Fig. 1 (b)], versus the magnetic field. For different magnetic fields, anti-crossings occur for different bias voltages. The black line and the squares describe a single-particle system, while the red line and the circles correspond to the many-particle $\nu=\frac{1}{3}$-FQHE system (Fig. 2). The single-particle anti-crossing gap is closed for the manyparticle system for $B \sim 8 \mathrm{~T}$. The number of electrons in the many-electron system is $N=9$ and the parameter of the sphere is $2 S=24$.

LL-2 has a maximum at the anti-crossing point, while the gap in LL-1 is suppressed. Therefore at the anti-crossing point the $\nu=\frac{1}{3}$-many-particle system is incompressible in LL-2 and compressible in LL-1. Since the incompressible FQHE state has a lower binding energy than that of the compressible state, this energy difference is enough to close the anti-crossing gap. Therefore, in a large magnetic field and as a function of the bias voltage, we should expect the following behavior: The FQHE system, which initially for a small bias voltage, $U<400 \mathrm{meV}$, is in LL-1, occupies LL-2 at the anti-crossing point, $U=400$ meV, leaving LL-1 empty. With further increase of the bias voltage, $U>400 \mathrm{meV}$, the system returns to LL-1, while LL-2 becomes empty. These transitions between different LLs at the anti-crossing point are accompanied by jumps in the value of the FQHE gap as illustrated by blue lines in Fig. 2 (d).

The strength of the FQHE, i.e., the magnitude of the FQHE gap, and correspondingly the cohesive energy of the FQHE states, is determined by the short-range properties of the interaction potential, i.e, the Haldane pseudopotentials at small values of the relative angular momentum, $m$. Therefore, at a weak magnetic field the short-range interaction strength at the anti-crossing point is enhanced in LL-1 (lower energy level), while for high magnetic fields the interaction strength at the anti-crossing point is increased in LL-2. This results in a weak enhancement of the many-particle anti-crossing gap for weak magnetic fields and strong suppression of the many-particle anti-crossing gap for strong magnetic fields. In Fig. 3, we present the anti-crossing gaps for single-particle and many-particle $\nu=\frac{1}{3}$-FQHE systems for different magnetic fields. For small values of the magnetic fields, $1.5 \mathrm{~T}<B<8 \mathrm{~T}$, the many-particle gap clearly shows an enhancement compared to that for the 
single-particle case. For $B \approx 8 \mathrm{~T}$ the many-particle anticrossing gap closes, and for $B>8 \mathrm{~T}$ the anti-crossing in a single-particle system becomes a double-crossing in the many-particle FQHE system.

It is known that the FQHE ground state of conventional semiconductor systems can be spin-polarized or spin-unpolarized. The polarization properties of the system are determined by the filling factor and strength of the applied magnetic field [11, 18]. While in conventional systems the $\nu=\frac{1}{m}$ FQHE state is always fully spin-polarized, for filling factors $\nu=\frac{2}{3}$ and $\nu=\frac{2}{5}$ there is a competition between the energies of spin-polarized and spin-unpolarized incompressible states [18]. With increasing strength of the magnetic field, the Zeeman energy of electrons favors the spin-polarized state, which results in possible spin-transitions in the system by varying the magnetic field. Those theoretical predictions subsequently received experimental confirmation [19, 22 25]. The TLG system shows a different type of spin transitions realized at the anti-crossing points, which could also be probed experimentally.

We have analyzed the spin properties of the FQHE states in TLG for filling factors $\nu=\frac{2}{3}$ and $\nu=\frac{2}{5}$. In Fig. 4 the results for $\nu=\frac{2}{3}$-FQHE state are shown for LL-1 and LL-2 without including the Zeeman energy. The black and red lines in Figs. $4(\mathrm{a}, \mathrm{b})$ correspond to spin-polarized and spin-unpolarized systems, respectively. The general behavior of the system is similar to that of the $\nu=\frac{1}{3}$-FQHE state. In a small magnetic field the system shows stronger interactions in LL-1, while for larger magnetic fields the anti-crossing of energy levels becomes double crossings. For a small magnetic field, the ground state of the $\nu=\frac{2}{3}$-FQHE system is mainly spin-polarized with only a small region of bias voltages, $U$, when the system becomes spin-unpolarized in LL-2. Therefore, for a weak magnetic field, $B \sim 5 \mathrm{~T}$, the $\nu=\frac{2}{3}$ FQHE system in LL-2 should show spin transition into an unpolarized state within a narrow interval of $U$ at the anti-crossing point. A strong Zeeman energy will however suppress this spin transition.

In a large magnetic field [Fig. 4 (b)], the $\nu=\frac{2}{3}$-FQHE system shows interesting spin properties. While in LL1 the $\nu=\frac{2}{3}$-FQHE ground state is spin-unpolarized for small values of $U$, it becomes spin-polarized at a large bias voltage, $U>420 \mathrm{meV}$. In LL-2 the ground state is spin-unpolarized for all values of $U$. Finally, combining these two behaviors and comparing the ground state energies of different systems [Fig. 4 (b)], we predict the following novel spin transitions. If the system is initially in LL-1 for the $\nu=\frac{2}{3}$-FQHE state, then with increasing bias voltage the system will undergo the following transitions: spin-unpolarized state in LL-1 $\Leftrightarrow$ spinpolarized state in LL-2 $\Leftrightarrow$ spin-unpolarized state in LL- $1 \Leftrightarrow$ spin-polarized state in LL-1. What is remarkable here is that, spin polarization of the $\nu=\frac{2}{3}$-FQHE system in TLG can be controlled by fine tuning the bias voltage - a possibility that never existed in the FQHE regime of conventional systems. We have also studied

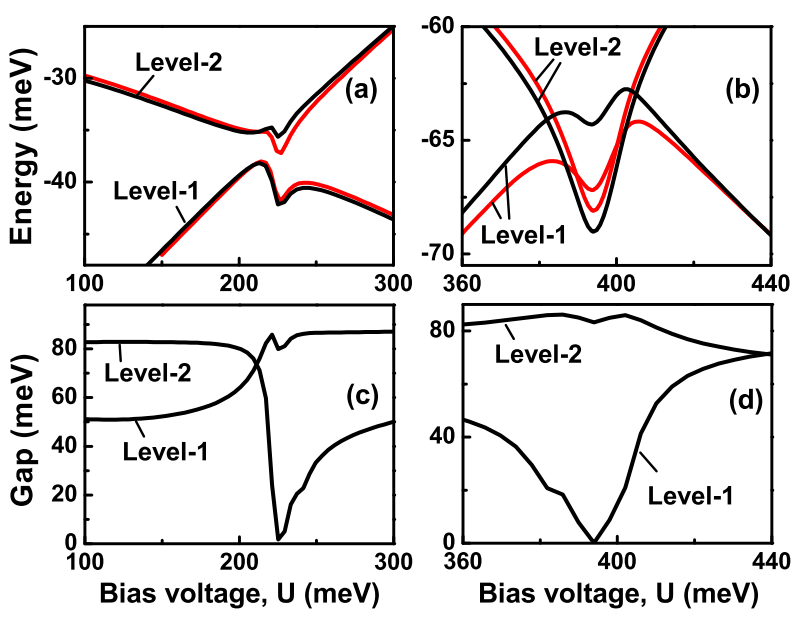

FIG. 4: The ground state energy per particle [panels (a) and (b)] and the excitation gaps [panels (c) and (d)] for $\nu=\frac{2}{3}$ FQHE in LL-1 and LL-2 versus the bias voltage near the anti-crossing points. The excitation gaps are shown only for the spin-polarized systems. The magnetic field is 5 Tesla [panels (a) and (c)] and 15 Tesla [panels (b) and (b)]. The black and red lines in panels (a) and (b) correspond to spinpolarized and spin-unpolarized systems, respectively. For the spin-polarized system, the number of electrons is $N=16$ and the parameter $2 S$ is 24 , while for the spin-unpolarized system $N=10$ and $2 S=12$.

the $\nu=\frac{2}{5}$ filling factor in LL-1 and LL-2. The general properties of the anti-crossing is similar to those of the $\nu=\frac{1}{3}$-FQHE system, i.e., an enhancement of the anti-crossing gap for a small magnetic field and strong suppression of the anti-crossing gap for a large magnetic field. However, for both LL-1 and LL-2 the $\nu=\frac{2}{5}$-FQHE ground state is spin-polarized, which excludes the possibility of spin transitions in that system.

In conclusion, trilayer graphene exhibits several unique electronic properties near the anti-crossing points of two Landau levels. In the FQHE regime, the electron-electron interaction strongly renormalizes the anti-crossing gap. In a weak magnetic field $(B<8 \mathrm{~T})$, the many-body interaction enhances the anti-crossing gap, resulting in a non-monotonic dependence of the excitation gaps on the bias voltage. In a large magnetic field $(B>8 \mathrm{~T})$, the electron-electron interaction strongly suppresses and finally closes the anti-crossing gap. In such large magnetic fields, the spin-polarized FQHE system shows nontrivial transitions as a function of the bias voltage, which are accompanied by jumps of the FQHE excitations gaps. In a large magnetic field $(B>8 \mathrm{~T})$ the TLG displays unique spin polarizations with controllable spin transitions. By varying the bias voltage, the $\nu=\frac{2}{3}$-FQHE system can be switched from spin-polarized to spin-unpolarized states. Various experimental techniques have been developed in the past to study spin transitions in the FQHE regime for conventional electron systems. These include measure- 
ments in a tilted magnetic field [19], application of hydrostatic pressure 22], optically pumped nuclear magnetic resonance 23], resistively detected nuclear magnetic resonance [24], time-resolved radiative recombination [25], among others. Similar experimental studies in trilayer graphene will undoubtedly uncover a wealth of information about charge and spin transitions revealed by a TLG in the FQHE regime.

The work has been supported by the Canada Research Chairs Program of the Government of Canada.
[ $\ddagger$ Electronic address: tapash@physics.umanitoba.ca

[2] K.S. Novoselov, Rev. Mod. Phys. 83, 837 (2011); A.K. Geim, ibid. 83, 851 (2011).

[3] D.S.L. Abergel, V. Apalkov, J. Berashevich, K. Ziegler, and T. Chakraborty, Adv. Phys. 59, 261 (2010).

[4] K.S. Novoselov, et al., Nature 438, 197 (2005); Y. Zhang, Y.-W. Tan, H.L. Stormer, and P. Kim, ibid, 438, 201 (2005).

[5] Z. Jiang, et al., Phys. Rev. Lett. 98, 197403 (2007); Y. Zhang, et al., Phys. Rev. Lett. 96, 136806 (2006).

[6] P.R. Wallace, Phys. Rev. 71, 622 (1947).

[7] K.S. Novoselov, et al., Nat. Phys. 2, 177 (2006).

[8] J.W. McClure, Phys. Rev. 104, 666 (1956); R.R. Haering and P.R. Wallace, J. Phys. Chem. Solids 3, 253 (1957).

[9] E. McCann and V.I. Fal'ko, Phys. Rev. Lett. 96, 086805 (2006); E. McCann, Phys. Rev. B 74, 161403 (R) (2006); J.M. Pereira, Jr., F.M. Peeters, and P. Vasilopoulos, Phys. Rev. B 76, 115419 (2007).

[10] D.C. Tsui, H.L. Störmer, and A.C. Gossard, Phys. Rev. Lett. 48, 1559 (1982); R.B. Laughlin, ibid. 50, 1395 (1983).

[11] T. Chakraborty and P. Pietiläinen, The Quantum Hall Effects (Springer, New York 1995) 2nd. edition.

[12] V.M. Apalkov and T. Chakraborty, Phys. Rev. Lett. 97, 126801 (2006).

[13] V.M. Apalkov and T. Chakraborty, Phys. Rev. Lett. 105, 036801 (2010); ibid. 107, 186803 (2007).

[14] X. Du, et al., Nature 462, 192 (2009); D.A. Abanin, et al., Phys. Rev. B 81, 115410 (2010); I. Skachko, et al., Phil. Trans. R. Soc. A 368, 5403 (2010).

[15] K.I. Bolotin, et al., Nature 462, 196 (2009); F. Ghahari, et al., Phys. Rev. Lett. 106, 046801 (2011).

[16] M. Koshino and E. McCann, Phys. Rev. B 83, 165443
(2011): S. Yuan, R. Roldan, and M.I. Katsnelson, Phys. Rev. B 84, 125455 (2011).

[17] T. Taychatanapat, K. Watanabe, T. Taniguchi, and P. Jarillo-Herrero, Nat. Phys. 7, 621 (2011); A. Kumar, et al., Phys. Rev. Lett. 107, 126806 (2011).

[18] T. Chakraborty, Adv. Phys. 49, 959 (2000); T. Chakraborty, P. Pietiläinen, and F.C. zhang, Phys. Rev. Lett. 57, 130 (1986); T. Chakraborty, Surf. Sci. 229, 16 (1990); T. Chakraborty and F.C. zhang, Phys. Rev. B 29, 7032 (R) (1984); F.C. Zhang and T. Chakraborty, ibid. 34, 7076 (1986); T. Chakraborty and P. Pietiläinen, ibid. 39, 7971 (1989); ibid. 41, 10862 (1990).

[19] R.G. Clark, et al., Phys. Rev. Lett. 62, 1536 (1989); J.P. Eisenstein, H.L. Störmer, L. Pfeiffer, and K.W. West, ibid. 62, 1540 (1989); A.G. Davis, et al., Phys. Rev. B 44, 13128 (1991); L.W. Engel, et al., ibid. 45, 3418 (1992); T. Sajoto, Y.W. Suen, L.W. Engel, M.B. Santos, and M. Shayegan, ibid. 41, 8449 (1990).

[20] F.D.M. Haldane, Phys. Rev. Lett. 51, 605 (1983).

[21] T. Chakraborty and V.M. Apalkov, in The Physics of Graphene, edited by H. Aoki and M. Dresselhaus (Springer, New York 2012) (to be published).

[22] W. Kang, et al., Phys. Rev. B 56, 12776(R) (1997); H. Cho, et al., Phys. Rev. Lett. 81, 2522 (1998).

[23] P. Khandelwal, et al. Phys. Rev. Lett. 81, 673 (1998); T. Chakraborty and P. Pietiläinen, ibid. 76, 4018 (1996); ibid., 83, 5559 (1999).

[24] L.A. Tracy, J.P. Eisenstein, L.N. Pfeiffer, and K.W. West, Phys. Rev. Lett. 98, 086801 (2007); O. Stern, et al., Phys. stat. sol. (b) 245, 428 (2008).

[25] I.V. Kukushkin, K. von Klitzing, and K. Eberl, Phys. Rev. B 55, 10607 (1997). 